\title{
Le « psychologisme » de Saussure à l'« âge psychologique » des sciences humaines
}

Original Study

\author{
Serge Tchougounnikov \\ Université de Bourgogne Franche-Comté, Laboratoire CPTC, France. \\ serge.tchougounnikov@yahoo.fr
}

Received: August 2021; Accepted: November 2021

\begin{abstract}
II s'agit de montrer que la linguistique saussurienne a maintenu les positions conventionnelles de la psychologie d’alors. Les développements de cette dernière permettent de mieux comprendre la visée du «psychologisme » de Saussure. Cet arrière-fond conceptuel explicite l'origine et la portée de certains concepts-clés saussuriens : l'arbitraire du signe linguistique ; le « signifiant » saussurien en tant « représentation du son »; la relation signifié/signifiant conçue comme « recto-verso d'une feuille de papier »; le modèle saussurien du signe linguistique. Ces éléments, déterminants pour sa portée et son influence, se rattachent aux développements de la psychologie de cette période : I'hétérogénéité constitutive du « Moi » tel qu'il est défini par Herbart et son école ; la distinction entre l'acte de représenter (Vorstellen) et l'objet représenté (Vorgestellt) conçus respectivement comme une détermination ontologique et comme expression de la représentation ; l'aperception ; la notion de « représentation verbale » (Wortvorstellung) telle qu'elle a été successivement élaborée par la psychologie de la conscience au cours du XIXème siècle et résumé par W. Wundt en 1900
\end{abstract}

Keywords: Saussure ; psychologisme ; arbitraire du signe linguistique ; signifiant ; aperception ; représentation verbale; valeur

On a beaucoup écrit sur le « psychologisme » saussurien. En résumant l'apport du «psychologisme » de Saussure à la linguistique, en particulier, par rapport aux positions des « néo-grammariens » allemands, Simon Bouquet formule ainsi l'ambiguité épistémologique du courant néo-grammarien : "Que l'objet épistémologique soit manqué par les néo-grammairiens, cela tient ici précisément à ce qu'ils définissent cet objet comme le produit d'un ordre hybride : à la fois ordre naturel (« physico-mécanique ») et ordre d'esprit («psychologique ») (Bouquet 1997, 93-94).

La complexité de l'évolution phonique serait tributaire de la complexité du psychisme qui dépasse le mécanisme de l'association analogique. S. Bouquet voit le problème épistémologique des néo-grammairiens dans l'absence « d'une théorie de l'esprit dans laquelle inscrire une théorie du langage tenant compte des résultats du comparatisme » (ibid., p. 95). Selon cet auteur, l'échec de la linguistique néo-grammairienne tiendrait donc au fait qu'elle « achoppe précisément sur une conception du matériel et du spirituel dans le langage » (ibid., p. 95)
Selon Bouquet, «l'essence même de la définition saussurienne de l'objet comparatiste - remettant en question l'ordre hybride qui prévaut dans la conception énoncée par Brugmann et Osthoff - se résume dans la thèse de la nature psychologique dudit objet » (ibid., p. 102). En fait $\mathrm{S}$. Bouquet y voit le passage de la vision hybride (= dualisme psychophysique) à une parfaite « digitalisation de l'esprit » (= vision symboliste ou cognitiviste) esquissée par Saussure. En effet, pour Bouquet, le postulat de la nature psychologique du langage se manifeste dans le fait, que sa vision « pose que la face phonologique de la langue appartient, tout autant que sa face sémantique, à l'ordre de l'esprit » (ibid., p. 102).

Le nouvel objet de la linguistique (assimilé à la « langue »), dont parle Saussure au début du Cours..., pourrait permettre de sortir du dualisme psychophysique, ce dogme absolu de la psychologie du 19ème siècle. Ainsi, la langue par sa spécificité même, dépasserait le caractère « hybride » de l'objet psychophysique. A partir de l'affirmation « tout est psychologique dans la langue » (ibid., p. 103), Saussure souligne la non-pertinence de 


\section{Le « psychologisme » de Saussure à l'« âge psychologique » des sciences humaines}

l'opposition : son (comme une unité mécanique, physique) versus analogie (phénomène irrégulier, psychique).

Ainsi, l'aspect mécanique et physique de l'évolution du langage (les lois phoniques) se trouve clairement opposé à l'aspect psychologique (défini comme « analogie»).

L’objet linguistique conçu par l'école néo-grammairienne surgit au croisement de ces deux dimensions. Dans cette optique les écarts par rapport aux lois phoniques (qui sont des lois physiques) sont soumis au psychisme par le mécanisme de l'analogie. Ces déviations sont des manifestations physiques du principe psychique inhérent à la conscience humaine. Le dualisme psychophysique, qui domine la psychologie allemande des années 1870-1880, se trouve donc maintenu au sein de ce modèle. En revanche, la nouveauté du projet de Saussure aurait consisté à remplacer le dualisme psychophysique caractéristique de la linguistique néogrammairienne par une prise de position anti-dualiste qui conduit à une «psychologisation » intégrale de l'objet de recherche linguistique.

Dans ce qui suit nous chercherons à montrer que la linguistique saussurienne a maintenu les positions conventionnelles de la psychologie d'alors. Qui plus est, les développements de la « linguistique psychologique » de cette période (dont celle des néogrammairiens) permettent de mieux comprendre la visée du «psychologisme» de Saussure.

En effet, cet arrière-fond conceptuel semble expliciter l'origine et la portée de certains concepts-clés de la linguistique saussurienne dans la mesure où ils révèlent leur ancrage dans les idées psychologiques d'alors. Les éléments essentiels, déterminants pour sa portée et son influence, se rattachent aux développements de la psychologie de cette période. II s'agit en particulier de la thèse de l'arbitraire linguistique qui est tributaire de plusieurs postulats psychologiques, parmi lesquels : I'hétérogénéité constitutive du « Moi » tel qu'il est défini par Herbart et son école ; la distinction entre l'acte de représenter (Vorstellen) et l'objet représenté (Vorgestellt) conçus respectivement comme une détermination ontologique et comme expression de la représentation; enfin, la notion de « représentation verbale » (Wortvorstellung) telle qu'elle a été successivement élaborée par la psychologie de la conscience au cours du XIXème siècle et résumé par W. Wundt en 1900. II s'agit en outre de la notion de « signifiant » conçu comme « image acoustique » ainsi que de l'idée du lien indissociable entre le signifié et le signifiant traduisible en langage psychologique comme conséquence de l'effet aperceptif.

Ainsi, cette mise en contexte de certains éléments psychologiques de la linguistique saussurienne cherche à contribuer à la « constitution d'un domaine de mémoire pour la linguistique moderne » (Chiss, Puech 1999, 53) et à la quête d' « une histoire de l'émergence disciplinaire » (ibid., p. 11) qui caractérise les études d'histoire et d'épistémologie des disciplines du langage (voir : Chiss, Puech 1997; Chiss, Puech 1999).

\section{LE DISPOSITIF DE L'ARBITRAIRE \\ AU SEIN DU PSYCHISME}

Curieusement, c'est le postulat de l'arbitraire du signe ${ }^{1}$ qui se laisse comprendre plus aisément par référence aux idées psychologiques de cette période. En effet, l'idée de l'« arbitraire du signe », ou encore du caractère arbitraire du lien entre l'idée et le son fait partie des affirmations de la psychologie du XIXème siècle. Rappelons que Saussure comprend par l'arbitraire ce qui « amorphe », « non-formé ». Pour lui, le terme d'arbitraire désigne l'«absence de forme » conçue comme propriété des unités linguistiques: " amorphe » signifie « ce qui n'a pas de forme en soi » (Godel 1957, 253).

Chez Saussure, l'arbitraire dépend des valeurs intrinsèques au système : la valeur «motivée » serait impossible car elle contiendrait alors un élément imposé au système du dehors. Or, pour Saussure, les valeurs émanent du système : «dans tous les cas nous surprenons au lieu d'idées données d'avance, des valeurs émanant du système » (Saussure [1916] 1969, 162). II convient de noter que pour les psychologues de cette période, et, en particulier, pour Wundt, les « représentations globales » (Gesamtvorstellungen) subissent, elles aussi, une différentiation à l'aide des éléments analytiques croissants (Wundt, 1900 B2, 234-247) assimilables aux « valeurs ».

En effet, aussi bien Saussure que Wundt utilisent le même argument d'une tendance analytique propre au psychisme. Pour Wundt, cette tendance se réalise comme l'ensemble des divisions à l'intérieur d'une « représentation globale » (sa vision de l'émergence des mots qui sont des fragments analytiques des phrases synthétiques). Pour Saussure, «la pensée, chaotique de sa nature, est forcée de se préciser en se décomposant », «la 'pensée - son' implique des divisions », « la langue élabore ses unités en se constituant entre deux masses amorphes » (Saussure [1916] 1969, 156). Saussure parle de «l'accouplement de la pensée avec ma matière phonique » (processus comparé à des vagues sur la surface d'eau : la décomposition de la surface en une série de divisions, c'est-à-dire de vagues » (Ibid., p. 156). Le fait linguistique ou la langue est « une série de subdivisions contiguës » sur deux plans indéfinis ou confus - des idées et des sons (Ibid., p. 155-156). Selon Saussure, " une idée se fixe dans un son » tout simplement parce que la langue « est le domaine des articulations » (Ibid., p. 156).

Saussure formule deux arguments pour fonder la nécessite de l'arbitraire linguistique : en premier lien, il s'agit du fait que le son et la pensée sont confus et amorphes; par conséquent, « le choix qui appelle telle tranche acoustique pour telle idée est parfaitement arbitraire » (Ibid., p. 157).

1 II s'agit de l'arbitraire du signe, compris comme « principe sémiologique par excellence » et qui « condense [...] tous les paradoxes de l'idée sémiologique » (Chiss, Puech 1992, 10). 


\section{Tchougounnikov}

En second lieu, pour Saussure, l'arbitraire linguistique est conditionné par les " valeurs » : le « tout solidaire», base entièrement relative et différentielle de tout système de signes; la valeur exprime la force du fait social, ou encore le fait social s'exprime par les valeurs, il crée le système linguistique. L'établissement des valeurs « dont I'unique raison d'être est dans l'usage et le consentement général » (ibid., 157), est tributaire de la communauté linguistique. On peut se demander, s'il s'agit là d'une autre solution de la question ethnopsychologique où le principe de la valeur viendrait remplacer le principe de « l'esprit du peuple »(Volksgeist). En effet, ces deux instances offrent une solution du dispositif de l'interaction collective dans la mesure où elles relèvent toutes les deux du fonctionnement des communautés linguistiques.

Nous verrons que la célèbre thèse de l'arbitraire du signe linguistique ${ }^{2}$ ne fait que reprendre le fait consensuel et largement établi par la psychologie de cette période. L'argumentation de l'ordre du « social vient rejoindre l'argumentation de l'ordre du «psychique».

\section{L'ARBITRAIRE AU SEIN DE LA REPRÉSENTATION}

On voit assez tôt surgir dans la psychologie du XIXème siècle l'idée de l'arbitraire comme étant constitutif du travail du psychisme. Pour Herbart, le sujet pris en tant que tel n'est rien dans la mesure où il n'est qu'une représentation (Vorstellen) de soi-même. Cet acte de représenter (Vorstellen) en tant qu'engendrement de l'image (Bild) doit, lui aussi, être une image représentée L'acte effectif de la représentation doit être une image de soi-même, une image qui, prise en tant que telle, n'est rien (Voir § 27 dans : Herbart, J.-F., [1824-1825] 1850).

C'est surtout la distinction herbartienne entre l'acte de représenter et le résultat de cet acte (Voir § $24-\S 27$; $\S 36$ dans: Herbart, J.-F., [1824-1825] 1850) qui pose l'arbitraire comme principe constitutif de la représentation. Pour Herbart, l'acticité du sujet dans l'acte de représenter reste inchangée ; en revanche, l'effet de cette activité, l'image représentée s'affaiblit ou disparaît. On y voit se manifester l'effet qui résulte de l'interaction de plusieurs représentations opposées (Voir § 24 § 25 § 26 dans : Herbart, J.-F., [1824-1825] 1850). Herbart appelle « visée » ou « tendance à représenter » (Streben) cet acte de représenter sans objets représentés (ou cette activité sans action accomplie ni effet produit). Si les représentations opposées sont réunies dans le même sujet qui doit accéder à la conscience de soi (ou encore atteindre le degré de conscience de soi), alors les représentations opposées s'inhibent mutuellement et se transforment en une tendance à représenter ( $\$ 24$ dans : Herbart, J.-F., [1824-1825] 1850).

Herbart envisage l'aspect quantitatif des représentations de deux points de vue: du point de vue de ce qui est représenté (objet de la représentation) et 2) du point de vue de l'acte de représenter (l'acte de la représentation). Pour Herbart, l'engendrement du « Moi » s'accompagne par la modification de la quantité des objets représentés ; mais l'acte de représenter reste non-modifié. Le sujet pris en soi-même est un acte de se représenter, de représenter soi-même, d'engendrer l'image de soi-même. L'acte de se représenter est l'image de soi-même qui, en tant qu'image, demeure sans pertinence. Ainsi, le fondement psychique du «Moi » (Ichheit) se compose d'éléments qui lui sont étrangers (Voir $\S 28$ dans : Herbart, J.-F., [1824-1825] 1850).

L'idée sous-jacente à cela consiste à dire qu'un acte représentationnel donné exprime par nature un certain contenu, toujours le même : dans ce cas, on voit s'établir entre les deux le lien intrinsèque. Cela ne veut pas dire pour autant que l'acte partage les qualités du contenu qu'il exprime (par exemple, le fait que le contenu d'une représentation spatiale soit étendu ne veut pas dire que la représentation en tant qu'acte soit elle-même étendue, ou encore, le fait que le contenu d'une représentation soit coloré comme c'est le cas de l'idée du rouge, ne signifie nullement que la représentation en tant qu'acte soit elle-même colorée). On y trouve par conséquent l'idée de signe comprise dans la définition de la représentation, même si en tant que telle la représentation est un signe transparent (une certaine représentation s'indique toujours elle même) $)^{3}$.

Herbart formule le principe général du mécanisme de la représentation comme suit : ce que nous représentons (ce qui est représenté par nous) doit nous déplacer de notre acte de représenter (ou de l'acte de représenter nous-mêmes). La possiblité même du « Moi » réside dans la suppression réciproque des représentations (Voir $\S 29$ dans : Herbart, J.-F., [1824-1825] 1850). Le « Moi » ainsi conçu apparaît comme une sorte de distorsion des contenus des représentation et comme le résultat d'une construction.

Le « Moi » ainsi conçu apparaît comme une sorte de distorsion des contenus des représentation et comme le résultat d'une construction.

Dans le domaine de la psychologie physiologique, les recherches de Hermann von Helmholtz accentuent dès 1855 « le caractère sémiotique de la perception » (En particulier, dans son traité Über das Sehen des Menschen [Sur la vue des hommes] (1855), voir : Wiesing 2002, 164).

Ainsi, dans son traité de 1878 Die Tatsachen der Wahrnehmung, Helmholtz formule un véritable argument psycho-physiologique de l'arbitraire dont le foyer serait le dispositif même des récepteurs. II écrit : « Nos sensations sont précisément des effets provoqués dans nos organes par des causes extérieurs; la façon, dont cet effet se manifeste, dépend naturellement en premier lieu de la nature de l'appareil sensoriel sur lequel il agit. Dans la

2 Voir aussi sur le problème de l'arbitraire et sa critique : Gandon 2006, 114-116; Suenaga 2005, 137-156.

3 Nous remercions de nouveau David Romand de sa communication personnelle relative à l'« arbitraire psychique » qui a donné un argument majeur aux présents développements. 
mesure où notre sensation nous apporte une information sur la nature de l'effet extérieur par lequel elle est produite, cette sensation peut être considérée comme un signe et non pas comme une image (ou encore, « comme un tableau de la chose », voire même « comme une représentation » : Abbild). En effet, on exige de l'image (Bild) une sorte de similitude avec l'objet représenté (abgebildet) : d'une statue une similitude de forme, d'un dessin - une similitude ou une analogie de la projection dans le champ de vision, d'un tableau - une similitude des couleurs. Or un signe n'a besoin d'aucune sorte d'analogie avec ce qu'il dont il est le signe. Les relations entre les deux se limitent au fait que le même objet appelle le même signe dans des circonstances semblables et que des signes différents entraînent toujours des effets différents » (Cité d'après: Wiesing 2002, 167-168).

L'idée de Helmholtz, selon laquelle les perceptions sensorielles sont autant de signes interprétés par le cerveau qui, par conséquent, reçoit des sens non pas les images des objets externes mais uniquement les signes, pose le problème essentiel de la neurophysiologie et jette les bases d'une théorie moderne de la connaissance. Elle introduit le niveau autonome des représentations entre I'homme et le monde et propose le modèle du cerveau conçu comme un dispositif qui représente, renvoie aux significations, travaille avec des symboles et engendre des représentations. Helmholtz en vient à l'idée du langage comme une clé possible de la question posée par l'idée du système nerveux interprétatif (Voir : Ruoff 2008, 20-21). C'est cette idée du langage comme représentant idéel de la pensée qui a inspiré le travail des linguistes de diverses orientations et qui finalement a joué un rôle éminent dans la résistance au programme behavioriste.

\section{LE « MOI » HÉTÉROGÈNE, FOYER DE L'ARBITRAIRE}

Cette idée du dispositif arbitraire au cœur même du psychisme reçoit une interprétation philosophique, elle est liée à la définition du « Moi » formulée par Herbart depuis 18164. Dans la perspective psychologique de cette période, l'arbitraire du signe découle, en premier lieu, des principes même du fonctionnement du psychisme ainsi que de la définition de la représentation qu'il implique. II s'agit d'une perspective philosophique dont cette psychologie est porteuse et qui est originellement inspirée par le kantisme.

Ce principe d'arbitraire est également lié à la délimitation proprement herbartienne entre la «statique » et la « dynamique de l'esprit » dans la mesure où Herbart différencie l' «Etre » et la représentation (Bild) (Herbart, J.-F., [1816] 1834, 64). Pour Herbart, les catégories de l'étant de s'applique pas à l' «Etre ». L'une des thèses essentielles du herbartianisme pose le phénomène comme n'étant pas identique avec la chose qui apparaît dans l'image. Pour Herbart, ce qui est pensé comme étant, s’appelle un « être ». Mais l'objet qui est détaché de l'être, l'objet pensé comme «quelque chose », gardera la désignation d'une «image» (Bild). En reprenant le point de vue kantien comme le point de départ de sa propre psychologie, Herbart se rapproche de la vision des sciences de la nature qui là où l'œil voit une couleur, considère qu'il y a des ondes électromagnétiques. Cela faisant, Herbart s'éloigne du courant phénoménologique en philosophie pour lequel la perception est la forme sous laquelle les choses elles-mêmes accèdent à la conscience. II s'agit pour Herbart de maintenir la statique de l'être à la différence de la dynamique des phénomènes. Herbart pose la notion d'image (Bild) et de formation (Bildung) comme des «formations internes » de la «substance simple » (Herbart, J.-F., [1816] 1834, 64-65). Ce sont des produits du système qui cherche à se conserver. Herbart souligne l'instabilité de la vie psychique. L'« arbitraire » dans la vie psychique est lié au clivage entre les propriétés sensibles des choses et des qualités réelles. C'est seulement dans leur apparition aux hommes qui les choses acquièrent leurs traits distinctifs (Voir § 16 dans : Herbart, J.-F., [1824-1825] 1850).

Ainsi, on voit se formuler l'idée d'une extrême spécificité des phénomènes psychiques. Dans cette vision, les éléments psychiques sont conçus comme des produits ou des constructions. La notion de «Moi » se construit dans la conscience du sujet pensant, elle résulte d'une décentralisation systématique qui se réalise à l'intérieur de chaque représentation (Voir sur la définition psychologique du Moi ainsi que sur sa composition et son fonctionnement : § 24 dans : Herbart, J.-F., [1824-1825] 1850).

Le «Moi » est un produit des relations qui s'établissent entre les représentations. Ce processus a pour corrélat une décentralisation à l'intérieur de chaque représentation. Le « moi » est un écart par rapport à ce qu'elles représentent initialement (voir § 24, § 25 dans : Herbart, J.-F., [1824-1825] 1850).

Ou encore : le «Moi » serait le résultat des modifications subies par chaque représentation isolée. Ce mécanisme psychique ébauche un système fermé autonome qui établit ses propres valeurs. Le «non-moi » des représentations se transforme en un Moi du sujet par la force du système psychique qui réalise une mise en relation des représentations.

Les relations isolées, entrant en relations, subissent des modifications quantitatives pour pouvoir constituer un « Moi ». L'effet de l'arbitraire au sein des représentations résulte en outre de l'affirmation de Herbart selon laquelle «l'âme n'est pas un fait de la conscience ». C'est pour cette raison qu'on peut appliquer le terme d' « arbitraire » ou de " hasard » au «Moi » (Ichheit). L' " âme » qui est définie comme « un substrat inconscient de la conscience de soi » est un épiphénomène (un accident) par rapport à ce « Moi » (Ichheit) (voir § 24 dans : Herbart, J.-F., [1824-1825] 1850).

Défini à travers la totalité des «masses aperceptive aperceptives préalables » constitutives du psychisme, le

4 En particulier, dans son Manuel de Psychologie (1816) et dans son traité Psychologie comme science (1824-1825). 
« Moi » de Herbart apparaît comme une hétérogénéité : il ne possède aucune unité réelle intrinsèque, elle est un conglomérat ou une multitude. C'est seulement dans l'introspection, dans le savoir interne subjectif du sujet particulier que ces « définitions individuelles » se composent en un sujet (voir § 25 dans : Herbart, J.-F., [1824-1825] 1850). Quand on se représente plusieurs objets, l'acte de les réunir dans une seule représentation se rapporte à celui qui se représente plusieurs objets hétérogènes, les réunit par son acte de représenter dans une seule représentation. C'est pourquoi, selon Herbart, ce qui est représenté par notre « Moi » doit nous écarter de nous-même pour que nous puissions arriver à nous-même (voir § 29 dans : Herbart, J.-F., [1824-1825] 1850). Pour Herbart, l'introspection (la perception interne) nous informe que nos sensations élémentaires forment diverses séries dont chacune comporte une multitude de représentations qui se caractérisent respectivement de divers degrés d'hétérogénéité (voir : ibid., § 36). L'« arbitraire psychique » est l'effet de l'absorption par « Moi » des éléments du monde externe. Cet effet est tributaire de la force assimilative et transformatrice de ces éléments par le «Moi ». Les éléments externes ne peuvent pas entrer dans la conscience de soi tels quels sous peine de faire disparaître la possibilité même de la conscience de soi : cette dernière serait simplement impossible. Si les représentations opposées se trouvent réunies dans le même sujet, sujet qui cherche à accéder à la conscience de soi, ces représentations doivent se transformer en une tendance à représenter, Streben (voir § 36. Le terme Streben signifie «tendre vers, s'efforcer de faire quelque chose, un effort vers quelque chose » (Herbart, J.-F., [1824-1825] 1850).

Le principe relationnel, que fait sien la psychologie herbartienne, contribue puissamment à l'affirmation de l'arbitraire au cœur du psychisme. C'est la relation qui devient l'unité essentielle de ce modèle du psychisme. Pour Herbart, l'âme est un " être simple », sans multiplicité dans sa qualité. On voir s'établir dans le psychisme une relation entre plusieurs « êtres simples », relation qu'on peut caractériser par une parabole du monde physique : pression et contre-pression. La coexistence des « réels » psychiques remplace la coexistence des constellations correspondantes de leurs interactions à l'état de tension face à leur contre tension. Tous les éléments de la vie psychique proviennent de l'interaction des représentations. Du fait que les représentations s'interpénètrent dans une seule âme, elles s'inhibent si elles s'opposent, et elles s'associent dans une force globale si elles ne sont pas opposées ${ }^{5}$.

L'arbitraire ainsi découvert par la psychologie d'inspiration herbartienne s'avère un phénomène purement psychique, un effet inévitable du régime même du psychisme, pour s'affirmer enfin dans le champ psychologique à titre d'axiome. Voilà pourquoi nous tendons à répondre à la question sur la nature de l'arbitraire : "L'arbitraire est-il 'philosophique' ? », posée en particulier par A. Suenaga (Suenaga 2005, 147) par : «L'arbitraire est psychologique ».

\section{LE SIGNIFIANT ET LA « REPRÉSENTATION VERBALE »}

Le modèle saussurien du signe comporte la célèbre notion de « signifiant » ou d'« image acoustique » qui fait partie de cette « entité psychique à deux faces » qu'est le signe linguistique (Saussure, [1916] 1969, pp. 98-99, 158-159). Pour Saussure, cette « image acoustique » ou encore cette « impression acoustique » ou ce « fait psychique » étranger à la physiologie, constitue une «impression existant de façon latente dans notre cerveau » (Godel 1957, 253). On sait que Saussure cherche à opposer le signifiant, cette « figure acoustique » ou encore cette « impression (ou image) acoustique » à l'acte articulatoire (ibid., p. 263). II convient de rappeler la note de Saussure sur le livre d'A. Sechehaye est à cet égard très significative. Ainsi, Saussure écrit à propos du passage où ce dernier se réfère aux observations pathologiques faites sur les diverses formes d'aphasie: " les cas d'aphasie montrent que la faculté de proférer [des sons] reste une chose distincte de la faculté d'évoquer les signes d'un langage régulier » (ibid., p. 51-52).

Le signifiant ou « image acoustique », ce constituant « sensoriel » du signe, défini aussi comme «l'empreinte psychique de ce son [matériel], la représentation que nous en donne le témoignage de nos sens », semble renvoyer à l'idée de « représentation verbale » (Wortvorstellung), conventionnelle aussi bien dans la linguistique psychologique que dans la psychologie de cette période. Pour les psychologues d'alors (et en particulier, pour W. Wundt) ${ }^{6}$, la « représentation verbale », qui médiatise les phénomènes du langage, est conçue comme un complexe associatif composé des éléments psychiques constitutifs des mots tels que: l'image sonore du mot, les sentiments articulatoires, le contenu sémantique.

Ainsi, le célèbre modèle sémiotique de Saussure opère une réduction du modèle psychologique traditionnel et n'en retient que deux éléments - l'image visuelle du mot, l'image sonore du mot (signifiant) et son contenu sémantique (signifié). D'ailleurs, Charles Bally et Albert Sechehaye, éditeurs du Cour..., se montrent parfaitement conscients de la généalogie du concept de « signifiant ».

5 Pour Herbart, ceci constitue la source de la volonté. La volonté n'est pas du tout primaire au sens d'une composante à priori du moi réflexif. Plus encore, c'est la somme des inhibitions liée à certaines représentations et les relations de ces inhibitions entre elles qui constituent la volonté comme une modification des processus des représentations (Herbart, [1816] 1834, 69-70).

6 Wundt expose cette notion, très courante en psychologie d'alors, en particulier dans ses Grundzüge der physiologischen Psychologie et les deux premiers volumes de Völkerpsychologie. 
Ainsi, ils ajoutent dans une note en bas de page à propos du terme d' « image acoustique »: « Ce terme d'image acoustique paraîtra peut-être trop étroit, puisqu'à côté de la représentation des sons d'un mot il y a aussi celle de son articulation, l'image musculaire de l'acte phonatoire. Mais pour F. de Saussure la langue est essentiellement un dépôt, une chose reçue du dehors [...] L'image acoustique est par exellence la représentation naturelle du mot en tant que fait de langue virtuel, en dehors de toute réalisation par la parole. L'aspect moteur peut donc être sous-entendu ou en tout cas n'occuper qu'une place subordonnée par rapport à l'image acoustique » (Saussure [1916] 1969, 98).

Il est à noter que pour conclure sur le « caractère psychique de nos images acoustiques », Saussure a recours à une expérience mentale qualifiable d'introspection : «Le caractère psychique de nos images acoustiques apparait bien quand nous observons notre propre langage. Sans remuer les lèvres ni la langue, nous pouvons nous parler à nous-même ou nous réciter mentalement une pièce de vers. C'est parce que les mots de la langue sont pour nous des images acoustiques qu'il faut éviter de parler des 'phonèmes' dont ils sont composés. Ce terme, impliquant une idée d'action vocale, ne peut convenir qu'au mot parlé, à une réalisation de l'image intérieure dans le discours » (ibid., p. 98)

Saussure a recours à la même expérience introspective que Steinthal en 1855 quand ce dernier cherche à prouver l'identité du langage et de la pensée. Or les résultats de ces deux introspections respectives sont opposés. Steinthal constate que l'acte de prononcer mentalement un discours ou un poème appris par cœur s'accompagne par des mouvements clairement perceptibles de la langue, qui sont autant de très faibles ébauches ou imitations des articulations. A l'appui de son expérience, Steinthal cite un passage de Herbart pour qui «la pensée silencieuse est en grande partie la parole retenue » : l'acte de penser s'accompagne des excitations nerveuses des organes de la parole qui est néanmoins trop faible pour déclencher le mouvement des muscles » (Steinthal 1855, 153). Cet exemple doit prouver l'affirmation de Steinthal selon laquelle le «son appartient à la pensée même » et lui est indispensable « comme le corps à l'esprit ». L'acte de penser sans paroles impliquerait l'acte de parler : « la pensée silencieuse est la parole pensée, la parole est la pensée énoncée » (ibid., p. 152-153).

En comparant ces deux expériences mentales il convient d'observer, que celle de Saussure se fonde sur l'exclusion de la composante psychophysique de l'articulation. Cette élimination concerne également les représentation correspondantes (image articulatoire, image graphique, image du mouvement associé à l'écriture du mot). Cette vision éliminatoire de la « représentations verbale » conditionne le modèle du signe saussurien à deux termes.

Ajoutons pour conclure : le « signifiant» saussurien dit aussi « image acoustique » est une traduction partielle du terme psychologique allemand de « représentation du son » (Lautvorstellung). L'effet proprement arbitraire, c'est-à-dire d'une relation «non-motivée» entre les constituants du signe, a pour base l'exclusion de la composante articulatoire, qui fait nécessairement partie du modèle psychologie du « mot » dit aussi « la représentation verbale ». II n'est guère étonnant que toute tentative de réintroduire la dimension motivée au sein du signe linguistique passe par la réactivation de cette instance articulatoire, de la représentation de l'articulation, exclue du modèle sémiotique de Saussure. La démarche de Jakobson est très caractéristique à cet égard (voir Jakobson, Waugh, 1980, 218-221).

\section{LE « RECTO-VERSO » DU SIGNE COMME EFFET APERCEPTIF}

La linguistique psychologique permet de contextualiser l'autre idée importante de Saussure, celle du lien indissociable entre la pensée et le son ainsi que le mécanisme saussurien des « articulations », illustré par la métaphore d' « une feuille de papier ». Rappelons que pour Saussure, « la langue est encore comparable à une feuille de papier : la pensée est le recto et le son est le verso; on ne peut découper le recto sans découper en même temps le verso; de même dans la langue, on ne saurait isoler ni le son de la pensée, ni la pensée du son; on n'y arriverait que par une abstraction dont le résultat serait de faire de la psychologie pure ou de la phonologie pure » (Saussure, [1916] 1969, p. 157). Cette « image saisissante» (Depecker 2009, 73) cherche à rendre compte de « la relation intérieur du signe avec l'idée » (Ibid., p. 74), en opposant au « son matériel » une nouvelle unité posée comme «le groupe son-idée » (Ibid., p. 75) ou comme « accouplement d'objets hétérogènes (signes-idées) » (Cité dans : Depecker 2009, 75). L. Depecker commente comme suit cette unité fondée sur la « relation intérieure » dans l'ordre de l'esprit : « Le 'groupe son-idée' : c'est-à-dire le son fusionné à l'idée en un groupe indissociable. C'est ce tout indissociable que forment «son » et « idée » qui, pour Saussure, constituent un « fait», une « identité linguistique » [...] Ce qui importe pour Saussure est donc l'association d'un son à une idée : le 'groupe son-idée', qui incorpore une idée dans un son et un son à une idée, rendant l'un et l'autre indissociable, mais sans consistance si on les prend séparément » (ibid., p. 75). Depecker perçoit cette défınition comme une rupture avec la tradition philosophique. II fait observer : « En distinguant deux côtés dans le signe et en affirmant leur caractère l'un à l'autre irréductible, mais indissociable, Saussure rompt avec la tradition philosophique » (Depecker 2009, 77).

Nous nous bornerons à ajouter que, du point de vue de la psychologie d'alors, il ne s'agirait guère d'une « rupture » : au contraire, pour les psychologues et les linguistes-psychologues de cette période la nature de cette

7 Rappelons que pour Saussure « chaque terme linguistique est un petit membre, un articulus où une idée se fixe dans un son et où un son devient le signe d'une idée » (Saussure, [1916] 1969, p. 156). 
« incorporation » et de cette « relation intérieure » se laisse décrire par l'idée hautement conventionnelle d'une « unité aperceptive » caractéristique du psychisme. En effet, pour tous les théoriciens de la « linguistique psychologique », il n'existe pas de différence fondamentale entre la forme du mot (le mot comme signe) et son contenu (contenu sémantique dans la mesure où ces deux éléments contribuent tous deux à la mise en œuvre de représentations.

C'est le mécanisme aperceptif élaboré par la psychologie de cette période qui fonde la métaphore d'une «feuille de papier » : en effet, selon la terminologie psychologique de l'époque, le contenu serait « aperçu » par le son, il s'agit donc de l'effet aperceptif entre la représentation sémantique et la représentation sonore (toutes deux constitutives de la « représentation verbale ») qui en fait « une entité psychique à deux faces », c'est-à-dire la représentation verbale abrégée de Saussure.

Le « son » conçu simultanément comme « représentation du son » et comme « représentation du mouvement articulatoire » appartient à la pensée et se donne comme un « objet mental ». II n'est donc nullement hétérogène à l'égard du psychisme et constitue un objet parfaitement psychologique et non-physiologique. Voila pourquoi, dans un autre contexte, l'affirmation des formalistes russes, selon laquelle le modèle du signe de Aleksandr Potebnia , concentré sur la « forme interne » du mot, néglige sa «forme externe », rate entièrement sa cible. Pour Potebnia, fondateur de la « linguistique psychologique » russe et partisan inconditionné de Steinthal et, par conséquent, herbartien, le «son matériel » ou la « forme externe » est un moyen d'aperception du concept et de l'ensemble de la «forme interne ». La distinction même entre la « forme externe » et la « forme interne » que cherche à souligner la critique formaliste, est de ce fait sans pertinence pour le modèle psychologique du signe verbal.

\section{LE MOT EN TANT QUE « REPRÉSENTATION VERBALE »}

En définissant le mot comme "association d'une impression acoustique et d'une idée » (Godel 1957, 253) Saussure reprend pour son compte le modèle que la psychologie de son temps a désigné par le terme de «représentation verbale» (Wortvorstellung). Cette approche analytique du psychisme se manifeste en particulier dans l'opposition établie par Saussure entre «impressions acoustiques » et «impression visuelles » (ibid., p. 253). C'est de la composition et du fonctionnement de cette dernière que résulte l'effet « arbitraire » du signe verbal, posé par la psychologie du XIXème siècle. Pour les psychologues d'alors, l'arbitraire du signe est garanti par le fait que les éléments psychiques associés sont différents au point de vue qualitatif. La signification verbale résulte de l'association arbitraire des images visuelles et sonores avec tel ou tel contenu représentationnel ainsi qu'avec les représentations articulatoires. Par conséquent, c'est le mode de cette liaison qui se place au centre des analyses. Ainsi, selon Wundt, la première question de l'étude psychologique des représentations verbales est la question du mode de liaison de leurs composantes (Voir le chapitre « La psychologie de la représentation. La structure psychique des représentations verbales » de sa Völkerpsycholgie dans: Wundt 1900, B1, 519). A cet égard, les troubles du fonctionnement du langage nous permettent de découper dans ses divers éléments les fonctions du langage en laissant en même temps reconnaître ces fonctions. Selon Wundt, «Ces troubles constituent pour nous la base la plus sûre de la structure psychique des représentations verbales » (ibid., p. 519).

Pour Wundt, ce que montrent ces troubles, c'est le fait que le mot est une figure psychique très composite (zusammengesetzt) qui, par sa nature complexe, est douée au plus haut degré de la capacité de transmettre des rapports associatifs dans les directions les plus différentes, ainsi que de se préserver soi-même contre les influences destructrices par des compositions entre ses éléments. Ainsi, à côté des sons de la langue, les sensations articulatoires représentent un élément constitutif qui n'est jamais absent mais qui, lorsque d'autres éléments sont inhibés, joue souvent un rôle prépondérant (ibid., p. 519). Ce sont aussi les signes écrits utilisés pour représenter un mot qui peuvent entrer dans ces associations et se trouver fortement reliés aux sensations articulatoires des organes du toucher qui accompagnent les mouvements de l'écriture.

La représentation verbale complète apparaît comme une « complication » (Complication) $)^{8}$ ternaire dont chacun des constituants est composé de deux éléments. Elle se compose de trois représentations complexes qui sont binaires : la « représentation du son » (Lautvorstellung), désigné par le symbole L et composé de l'élément acoustique (a) de celle-ci ainsi que de l'élément moteur ( $m$ ) de la « sensation articulatoire» (Articulationsempfindung); la « représentation du signe verbal » (Zeichenvorstellung ou Wortzeichen), désigné par le symbole Z et composé de l'élément optique (o) du mot-signe (Wortzeichen) ainsi que de l'élément moteur $\left(\mathrm{m}^{\prime}\right)$ de la « sensation du mouvement » ou « sensation motrice » (Bewegungsempfindung) ; la « représentation conceptuelle » (Begriff ou Begriffsvorstellung) qui se décompose, elle aussi, en deux éléments : la représentation objective ( $v$ = objective Vorstellung) et la tonalité émotionnelle qui l'accompagne ( $\mathrm{g}$ = begleitende Gefülston) (ibid., p. 520).

Au sein de cette « complication » ou « combinaison complexe », l'on doit penser chaque membre lié à chaque autre de sorte qu'il puisse devenir efficace (wirksam) en partie directement et en partie indirectement comme l'aide à l'association. En outre tout composant peut perdre toutes ses associations ou seulement

8 Wundt utilise le terme harbartien censé rendre compte du fonctionnement des représentations, qui pourrait être aussi rendu par « combinaison complexe» (ibid., p. 520). 
certaines parmi elles. Enfin, une association peut être totalement détruite; dans ce cas la disparition de ce lien peut être compensée par des aides associatives qui deviennent progressivement efficaces par l'entraînement (ibid., p. 520).

Il s'agit de la dépendance de la combinaison complexe verbale à l'égard de la fonction elle-même ainsi qu'à l'égard des aides associatives, qui se produisent lorsque certaines orientations fonctionnelles disparaissent et qui sont consolidées par exercice et par les aides associatives. Cette dépendance a pour conséquence le fait que les différentes liaisons ou associations dont se compose une association verbale complète présentent, selon les cas, des relations entre elles variables (ibid., p. 524-525).

Le caractère déterminant de l'association consiste en ce qu'elle est une association simultanée. Elle est simultanée parce que la représentation particulière qui en résulte est perçue simultanément dans toutes ses parties. C'est en cela que ses parties deviennent les traits distinctifs (Merkmale) psychologiques. La représentation verbale est une association car elle ne comporte pas d'autres liaisons que celles qui se produisent dans tout autre type d'association. Pour Wundt, il s'agit de processus élémentaires et non pas de « processus de masse ». Quand Herbart et les herbartiens désignent ces associations comme effets des masses aperceptives, cette expression induit en erreur de double manière : 1) parce qu'elle sépare les processus des associations (auxquelles elles appartiennent par leur nature) et 2) parce que tout ce processus est exactement le contraire de l'effet de masse (ibid., 542-543).

Pour Wundt, un mot connu est aperçu en général directement comme un tout qui forme une unité, il n'est pas recomposé par la suite dans notre représentation à partir de ses composantes. Au contraire, nous percevons des lettres et des sons non pas comme des parties mais comme des caractéristiques (Merkmale) du tout. Ce n'est que le mot qui dépasse les limites de la capacité de la perception qui devient l'objet d'une aperception successive (ibid., p. 534).

Ainsi, Wundt conçoit la représentation verbale comme un système psychique dynamique dont les éléments et les liens associatifs sont perpétuellement renouvelés en fonction de l'évolution du discours. Diverses composantes de la représentation verbale - les images visuelles, les images sonres, les sentiments d'articulation et le contenu sémantique - s'expriment avec plus ou moins d'intensité selon que le mot est prononcé, entendu, lu ou écrit. II appartient à l'aperception, c'est-à-dire au sentiment d'activité et de passivité lié à l'écoulement de nos représentations, de faire ressortir certaines représentations verbales ou certains éléments de la représentation verbale, c'est-à-dire de les faire accéder au champ clair de la conscience (Romand, Tchougounnikv 2011, 533-534).

\section{DE LA « SCIENCE SOCIALE »À LA "SCIENCE DES VALEURS "}

C'est ainsi qu'on voit émerger dans le champ psychologique l'idée de l'arbitraire constitutif du psychisme dans la mesure où l'on voit s'affirmer la « représentation verbale », concept banal à l'époque. Ce concept multiplie les liens formels et qualitatifs entre les éléments constitutifs de la représentation sensorielles (image visuelle du mot, représentation du son, sensations articulatoire) et représentation signifiante (Begriffsvorstellung). Enfin, il ne reste plus qu'un lien associatif donc fonctionnel entre les deux. Ce dispositif « arbitraire » constitutif du psychisme semble suggérer que le principe de l'arbitraire est parfaitement compatible avec l'approche cognitiviste.

Pour comprendre en termes psychologiques la question du rapport entre le signifiant et le signifié, tel qu'il avait été posé par la psychologie du XIXème siècle, il convient de se rappeler de nouveau l'importance de la différence entre l'acte de représenter (Vorstellen) et le contenu de la représentation (Vorgestellte) initialement issue de la psychologie de Herbart. II s'agit de délimiter le fait que la représentation existe effectivement et le fait que l'acte représentationnel exprime certaine qualité phénoménale ou sémantique. Cette délimitation entre l'acte de représenter (la détermination ontologique de la représentation) et l'objet représenté (l'expression du psychisme) introduit la dimension sémiotique au sein même de la représentation et en fait l'une des déterminations du fonctionnement psychique (Voir : § 24-26 et $\S 36$ dans : Herbart, J.-F., [1824-1825] 1850).

On retrouve le même modèle psychologique dans les développements de Wundt à propos de l'évolution du langage gestuel, en particulier, dans le cadre du passage progressif des gestes indicatifs aux gestes symboliques (ces derniers étant analogues sémiotiquement aux mots du langage articulé). Dans le geste indicatif, le représenté et le représentant se confondent, le signe s'indique lui-même comme étant son propre signifié (il s'agit d'une sorte de «signe transparent »). En d'autres termes, dans ce cas précis l'acte de représentation est le signe de son propre contenu. En revanche, dans le geste symbolique la représentation qui signifie (geste en tant qu'il est perçu) est interprétée non pas sur la base de son propre contenu, mais à partir du contenu d'une autre représentation (plus abstraite) qui n'a rien à voir avec elle sinon qu'elle est reliée à elle par association. Dans ce cas, on a une hétérogénéité qualitative ou formelle entre le signe et ce qu'il signifie. Ainsi, il n'y a pas aucune raison intrinsèque qui fasse que la représentation $A$ signifie la représentation B (Wundt 1900, B1, 149-187). Selon Wundt, l'évolution du langage gestuel s'accomplit sous forme de passage des « gestes déictiques » et « imitatifs » (die hinweisenden und die nachahmenden Geberden) vers les « gestes symboliques » (die symbolischen Geberden). II retient et analyse trois types fondamentaux des gestes: " gestes imitatifs » (nachbildenden); " gestes d'accompagnement co-désignatifs » (mitbezeichnenden) et les « gestes symboliques » (symbolischen ») (ibid., 151). 


\section{Tchougounnikov}

Il n'en demeure pas moins que la démarche de Saussure s'écarte du principe de l'« arbitraire psychologique» largement conventionnel à l'époque au nom d'un autre dispositif défini comme «valeur ». Chez Saussure, la « valeur » ne cesse pas pour autant d'être un phénomène psychique dans la mesure où elle se trouve reliée à la représentation. II écrit en effet : « quand on parle de la valeur d'un mot, on pense généralement et avant tout à la propriété qu'il a de représenter une idée, c'est là en effet un des aspects de la valeur linguistique » (Saussure [1916] 1969, 158). II s'avère en outre que l'arbitraire est lié à la propriété différentielle : « arbitraire et différentielle sont deux qualités corrélatives » (ibid., p. 163). Ainsi, la « valeur » saussurienne semble être une nouvelle manifestation du phénomène psychologique défini par la psychologie d'alors en termes de «tendance analytique » du psychisme.

On se souvient que Saussure récuse tout recours à la psychologie au nom des « sciences sociales $»^{9}$ qui étudient les « valeurs » et qui ont besoin des « concepts spécifiques», c'est-à-dire au nom de la spécificité du «fait linguistique et grammatical » (Godel 1957, 52) Ainsi, Saussure proteste contre la réduction des « sciences sociales » à la psychologie : «Car il faut « poser le fait grammatical en lui-même et dans ce qui le distingue de tout autre acte psychologique ou [...] logique » (ibid., p. 52). Pour définir le fait linguistique dans son autonomie, il faut, selon Saussure, «fixer le champ de l'expression et d'en concevoir les lois, non dans ce qu'elles ont de commun avec notre psychisme en général, mais dans ce qu'elles ont au contraire de spécifique et d'absolument unique dans le phénomène de la langue » (Cite dans : ibid., p. 52)

La même exigence de la spécificité du « fait linguistique et grammatical » ressurgit au sein du courant formaliste en Russie. On reconnait dans cette prise de position la quête formaliste d'une «science autonome » du langage et de la littérature (formulée aussi bien en Russie qu'en Allemagne). Le principe d'anti-psychologisme caractérise à la fois le principe émanant de l'idée des « sciences sociales » et le principe formaliste de la « forme étudiée en elle-même et pour elle-même ». Dans les deux cas il s'agit de fonder un nouveau projet scientifique sur le principe des « valeurs » émanées par le système lui-même que ce soit le système « social » ou « psychique ».

\section{BIBLIOGRAPHIE}

Bouquet, S., 1997. Introduction à la lecture de Saussure. Paris : Payot \& Rivages.

Chiss, J.-L., Puech, Ch., 1992. «Signe et langue : idée, projet, point de vue sémiologique ». In Puech, Ch. (éd.),
Sémiologie et histoire des théories du langage. Langages, $n^{\circ} 107$, Paris : Larousse, 6-27.

Chiss, J.-L., Puech, Ch., 1999. Le langage et ses disciplines XIXe - XXe siècles. Paris, Bruxelles: De Boeck $\&$ Larcier, Duculot.

Depecker, L., 2009. Comprendre Saussure d'après les manuscrits. Paris : Armand Colin.

Gandon, F., 2006. Le nom de l'absent. Epistémologie de la science saussurienne des signes. Limoges: Lambert-Luca.

Godel, R., 1957. Les sources manuscrites du Cours de linguistique générale de F. de Saussure. Genève : Dror, Paris : Minard.

Herbart, J.-F., [1816] 1834. Lehrbuch zur Psychologie. Königsberg, (Nachdruck der erste Ausgabe, Amsterdam : E. J. Bonset, 1965).

Herbart, J.-F., [1824-1825] 1850. „Psychologie als Wissenschaft". In Herbarts sämtliche Werke, B. 5, Schriften zur Psychologie. Leipzig: Verlag von Leopold Voss.

Jakobson, R., Waugh, L., 1980. La charpente phonique du langage. Paris : Minuit.

Romand, D., Tchougounnikov, S., 2009. « Aux origines allemandes du cognitivisme, introduction ». In Romand, D., Tchougounnikov, S. (eds.). Psychologie allemande et sciences humaines en Russie: anatomie d'un transfert culturel (1860-1930). Revue d'Histoire des Sciences Humaines 21, 3-27.

Romand, D., Tchougounnikov, S., 2010. «Le formalisme russe, une séduction cognitiviste ». In Berelowitch, W., Espagne M. (eds.), Sciences humaines et sociales en Russie à l'Âge d'argent : quelques figures de transferts, Cahiers du Monde russe, n 51/4, 521-546.

Ruoff, M., 2008. Hermann von Helmholtz. München : Fink.

Saussure, F., [1916] 1969. Cours de linguistique générale. Paris, Payot.

Steinthal, H., 1855. Grammatik, Logik und Psychologie. Ihr Prinzipien und ihr Verhältnis zu einander, Berlin : Fer. Dümmler's Verlagsbuchhandlung.

Suenaga, A., 2005. Saussure, un système de paradoxes. Langue, parole, arbitraire et inconscient. Limoges : Lambert-Luca.

Wiesing, L. (éd.), 2002. Philosophie der Wahrnehmung. Modelle und Reflexionen. Frankfurt am Main : Suhrkamp.

Wundt, W., [1874] (1908, 1910, 1911). Grundzüge der physiologischen Psychologie, 3 vol.. Leipzig: Engelmann.

Wundt, W., 1900. Völkerpsychologie. Eine Untersuchung der Entwicklungsgesetze von Sprache, Mythos und Sitte, Bd. 1, Bd. 2. Leipzig: Verlag von W. Engelmann.

Return to front page $\uparrow$

9 On se souvient que la distinction entre, d'un côté, le « fait individuel » et, de l'autre, le « fait social » chez Saussure demeure hésitante, ce qui aboutit parfois aux formulations contraires : «La langue est le réservoir individuel ; tout ce qui entre dans la langue, c'est-à-dire dans la tête, est individuel » et « tout ce que l'on considère en effet dans la sphère intérieure de l'individu [= la langue !] est toujours social... » (cité dans : Suenaga 2005, 34-35). Voir en particulier sur les «fluctuations dans la façon qu'a Saussure d'attribuer les catégories 'social' et 'individuel' aux termes de 'langue' et de parole' » : (ibid., p. 35). 